\title{
Dielectric response of modified Hubbard models with neutral-ionic and Peierls transitions
}

\author{
Zoltan G. Soos, Sharon A. Bewick \\ Department of Chemistry, Princeton University, Princeton \\ Andrea Peri, Anna Painelli \\ Dip. di Chimica GIAF Università di Parma, and INSTM-UdR Parma, 43100 Parma, Italy
}

(Dated: November 18, 2018)

\begin{abstract}
The dipole $P(F)$ of systems with periodic boundary conditions (PBC) in a static electric field $F$ is applied to one-dimensional Peierls-Hubbard models for organic charge-transfer (CT) salts. Exact results for $P(F)$ are obtained for finite systems of $N=14$ and 16 sites that are almost converged to infinite chains in deformable lattices subject to a Peierls transition. The electronic polarizability per site, $\alpha_{e l}=(\partial P / \partial F)_{0}$, of rigid stacks with alternating transfer integrals $t(1 \pm \delta)$ diverges at the neutral-ionic transition for $\delta=0$ but remains finite for $\delta>0$ in dimerized chains. The Peierls or dimerization mode couples to charge fluctuations along the stack and results in large vibrational contributions, $\alpha_{v i b}$, that are related to $\partial P / \partial \delta$ and that peak sharply at the Peierls transition. The extension of $P(F)$ to correlated electronic states yields the dielectric response $\kappa$ of models with neutral-ionic or Peierls transitions, where $\kappa$ peaks $>100$ are found with parameters used previously for variable ionicity $\rho$ and vibrational spectra of CT salts. The calculated $\kappa$ accounts for the dielectric response of CT salts based on substituted TTFs (tetrathiafulvalene) and substituted CAs (chloranil). The role of lattice stiffness appears clearly in models: soft systems have a Peierls instability at small $\rho$ and continuous crossover to large $\rho$, while stiff stacks such as TTF-CA have a first-order transition with discontinuous $\rho$ that is both a neutral-ionic and Peierls transition. The transitions are associated with tuning the electronic ground state of insulators via temperature or pressure in experiments, or via model parameters in calculations.
\end{abstract}

\section{INTRODUCTION}

Torrance et al $\stackrel{1}{n}$ reported the first neutral-ionic transition (NIT) in the organic charge-transfer (CT) salt TTFCA. Tetrathiafulvalene (TTF) is a potent $\pi$-electron donor (D), while chloranil (CA) is a strong $\pi$-acceptor (A). As confirmed subsequently by detailed vibrational and structural studies $\stackrel{2.3 .4}{=}$ the $300 \mathrm{~K}$ structure contains mixed regular stacks ... $\mathrm{D}^{\rho+} \mathrm{A}^{\rho-} \mathrm{D}^{\rho+} \mathrm{A}^{\rho-} \ldots$ with face-toface overlap and ionicity $\rho \sim 0.3$. Equal intermolecular interaction with each neighbor follows from inversion symmetry at the centers of TTF and CA. Below the firstorder transition at $\mathrm{T}_{c}=81 \mathrm{~K}$, the dimerized ionic stacks have $\rho \sim 0.7$ and alternately short and long intermolecular spacing. The ionic lattice is unstable to a transition that becomes a spin-Peierls transition ${ }^{\underline{5}}$ in the limit $\rho \rightarrow$ 1. In TTF-CA and other CT salts with discontinuous $\rho$ at $\mathrm{T}_{c}$, the neutral-ionic and Peierls transitions coincide.

Systems with neutral-ionic or valence transitions are necessarily rare: two ground states (GS) with different charge densities must be almost degenerate in order to switch between them by changing temperature or pressure. Horiuchi et al. 6.7.8.9.10.11 have recently studied NITs in a series of substituted TTFs and CAs whose static dielectric responses have large peaks $(\kappa>100)$. Their interpretation is a quantum phase transition between a neutral-regular and an ionic-dimerized GS controlled by an external parameter such as temperature or pressure ${ }^{11}$ No modeling of $\kappa$ is proposed, however, and no modeling is possible without the static polarizability of extended systems. In this paper we compute the static polarizability of Peierls-Hubbard models for organic CT salts and vary GS parameters to induce transitions. We obtain explicitly electronic and vibrational contributions to $\kappa$ for correlated states. Systems with continuous $\rho$ at the $\kappa$ peak and $\rho<1 / 2$ throughout are of particular interest, since they do not fit the simple paradigm of neutral-regular or ionic-dimerized.

The dielectric constant, $\kappa$, is the GS polarizability $\alpha$, or induced dipole moment, per unit cell in the crystal. The modern theory of polarization in insulators 12 has resolved the problem of a position or dipole operator in systems with periodic boundary conditions (PBC). Resta 13 introduced the following expression for the expectation value of the dipole moment, $P$ :

$$
P=\frac{1}{2 \pi} \operatorname{Im} \ln \left\langle\psi\left|\exp \left(i \frac{2 \pi M}{N}\right)\right| \psi\right\rangle \equiv \frac{1}{2 \pi} \operatorname{Im} \ln Z
$$

Here $|\psi\rangle$ is the exact electronic GS, $M$ is the conventional dipole operator and $\mathrm{PBC}$ are ensured via supercells. Polarization in insulators is related to the phase rather than the amplitude of $\psi$ and can be formulated as a Berry phase. $P$ applies to correlated as well as noninteracting systems and provides a unified approach to previous treatments of polarization and GS localization in insulators $\stackrel{14}{=}$ The magnitude of $Z$ is finite in insulators and zero in conductors. The phase or twist operator in Eq. (1) also appears in other contexts $\frac{15}{15}$ We will work with $P$ for the exact GS of modified Hubbard models with $N$ sites.

Although a static electric field $F$ is not compatible with PBC, Nunes and Gonze ${ }^{16}$ showed recently how to 
combine $F$ and $P$. They propose to define $P(F)$ with $\psi(F)$ in Eq. (11) and to minimize a functional that, in a one-dimensional system, is

$$
E\left(\psi_{F}, F\right)=E\left(\psi_{F}\right)-N F P\left(\psi_{F}\right)
$$

Several groups ${ }^{17,18,19,20}$ have applied Eq. (2) to the electronic dielectric constants of metal oxides and silicates, using Wannier functions for $\psi(F)$ and perturbation theory to find corrections to $\psi(0)$. As shown below, correlated models with finite $N$ are simpler because the basis is finite, which allows exact solution for $\psi(F)$ in Eq. (2). The procedure in Section 2 differs from the model-exact treatment of static or dynamic nonlinear op-

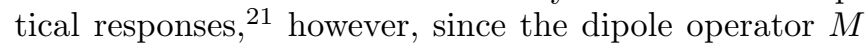
is now in the exponent.

The optical phonon of the mixed regular stacks .. $\mathrm{D}^{\rho+} \mathrm{A}^{\rho-} \mathrm{D}^{\rho+} \mathrm{A}^{\rho-} \ldots$ describes a rigid displacement of either sublattice, and in the hypothesis of linear electronphonon (e-ph) coupling leads to alternating CT integrals: $t(1+\delta), t(1-\delta)$. We assign the optical phonon a harmonic potential with frequency $\omega_{P}$ at $\rho=0$. With increasing $\rho$, e-ph coupling generates large anharmonicities ${ }^{22}$ and an overall softening of the vibrational frequency, which vanishes at the Peierls transition. A static electric field, $F$, strongly affects the lattice degrees of freedom at intermediate $\rho$, especially near the Peierls transition. The polarizability per site has then substantial electronic and vibrational contributions,

$$
\frac{d P}{d F}=\frac{\partial P}{\partial F}+\frac{\partial P}{\partial \delta} \frac{\partial \delta}{\partial F}=\alpha_{e l}+\alpha_{v i b}
$$

The $P$ derivatives above illustrate that physical processes are associated with changes 14 of $P$ and underscore the central role of $P$ for the dielectric response. In the vibrational part, $\frac{\partial P}{\partial \delta}$ is related to the IR intensity of the Peierls $\operatorname{mode}^{22}$ and $\frac{\partial \delta}{\partial F}$ to the field-induced change in dimeriza-

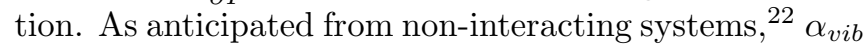
has a peak at the Peierls transition that is further enhanced by the frequency softening. The electronic part, by contrast, diverges at the NIT of the rigid regular lattice but varies slowly near the Peierls instability of deformable lattices.

The paper is organized as follows. Section 2 presents the exact solution of $P(F)$ using Eq. (2) for quantum cell models with a large but finite basis. The electronic polarizability of modified Hubbard models with fixed alternation is obtained in Section 3. The roles of lattice vibrations are introduced in Section 4 and developed explicitly for the dimerization mode and its softening at the Peierls transition. Section 5 contains polarizability, ionicity and related results for Peierls-Hubbard models with equilibrium dimerization. The $\kappa$ peak at the Peierls transition is due to $\alpha_{v i b}$ and increases with the lattice stiffness. We discuss in Section 6 the applicability of model results to dielectric peaks in organic CT salts.

\section{STATIC ELECTRONIC POLARIZABILITIES}

Quantum cell models typically have a single Wannier orbital $\phi_{p}$ per site that, in principle, can incorporate intra-site correlations ${ }^{23}$ On-site repulsion $U>0$ is kept in Hubbard models and long-range Coulomb interactions in the Pariser-Parr-Pople model ${ }^{24}$ Total spin $S$ is usually conserved, as in models for CT salts, but this condition can be relaxed. The defining feature of cell models is a large but finite many-electron basis in real space that is complete for a finite number of orbitals $\phi_{p}$. Slater determinants of $\phi_{p}$ 's can be used in general for eigenstates of $S_{z}$, but linear combinations of determinants are required for the smaller basis when $S$ is conserved. Easily visualized valence bond $(\mathrm{VB})^{25}$ diagrams are then convenient and used below.

The normalized singlet $(S=0)$ diagram $|k, q\rangle$ specifies the occupation number $n_{p k}=0,1$ or 2 at each site $p=1,2, \ldots N$ and the spin pairing of all singly-occupied site. The index $q$ is introduced below for the eigenvalue $M_{k}$ of the dipole operator. The VB basis is orthogonal with respect to charge, but there is overlap $S_{k k^{\prime}}$ between diagrams with identical $n_{p k}$ and different spin pairing. The operator $M$ for $N$ sites with uniform spacing $a=1$ and $e=1$ is

$$
M=\sum_{p=1}^{N} p q_{p}=\sum_{p=1}^{N} p\left(z_{p}-n_{p}\right)
$$

Here $q_{p}=z_{p}-n_{p}$ is the charge operator, with $z_{p}=0$ at $\mathrm{A}$ and $z_{p}=2$ at $\mathrm{D}$, and there are two electrons per DA unit cell. The neutral GS has regular spacing $a$ along the stack while the dimerized ionic GS has alternating spacing $a \pm u$. For simplicity we use $M$ in Eq. (44) for both regular and dimerized GS, thereby neglecting the motion of $\pm e \rho$ charges; corrections for $\pm u$ can readily be included $\stackrel{22}{=} M$ is diagonal in the $|k, q\rangle$ basis. Its eigenvalues are the integers, modulo $N, M_{k}=0, \pm 1, \pm 2, . .,-N / 2$. We take them as $q=2 \pi M_{k} / N$ for $-\pi \leq q<\pi$ in the first Brillouin zone. The matrix element in Eq. (11) is then ${ }^{22}$

$$
\left\langle\psi\left|\exp i \frac{2 \pi M}{N}\right| \psi\right\rangle=\sum_{q} W_{q} \exp i q \equiv Z_{x}+Z_{y}
$$

where $W_{q} \geq 0$ is the weight of basis vectors $|k, q\rangle$ with $q=2 \pi M_{k} / N$. Diagrams with $q \sim 0$ have greatest weight on the neutral side and lead to $Z_{x} \sim 1$, while diagrams with $q \sim \pm \pi$ and $Z_{x} \sim-1$ dominate on the ionic side. The regular chain has real $Z$ by symmetry and $Z=0$ at the NIT.

Since the VB basis is complete, the GS can be expanded as

$$
\psi(F)=\sum_{k, q} c_{k q}(F)|k, q\rangle
$$

The linear coefficient $c_{k q}$ are real and known at $F=0$ by hypothesis. Substitution of Eq. (6) into Eq. (2) and 
varying the $c_{k q}$ leads to a secular determinant. As discussed elsewhere $\stackrel{25}{25}$ sparse-matrix methods that exploit completeness are much more convenient for large $\left(>10^{6}\right)$ basis sets. We consequently seek an operator whose matrix elements correspond to $P(F)$ in Eq. (2). The partial derivative of $P(F)$ with respect to $c_{k^{\prime} q}$ is

$$
\frac{\partial P(F)}{\partial c_{k^{\prime} q}}=\sum_{k, q} c_{k, q}(F) S_{k^{\prime} k}\left\{\frac{Z_{x}(F) \sin q-Z_{y}(F) \cos q}{2 \pi\left[Z_{x}(F)^{2}+Z_{y}(F)^{2}\right]}\right\}
$$

We define the quantity in $\{\ldots\}$ as $\mu_{k q}$ and interpret it as the induced dipole of diagram $|k, q\rangle$. Since precisely the same result is obtained by introducing diagonal energies $-N F \mu_{k q}$ for each diagram, the desired induced-dipole operator is

$$
\Delta M(F)=\frac{N}{2 \pi} \operatorname{Im} \frac{\exp (i 2 \pi \hat{M} / N)}{Z(F)}
$$

It follows immediately that $|k, q\rangle$ is an eigenfunction of $\Delta M$ with eigenvalue $N \mu_{k q}$, as given by Eq. (7). The large response or polarizability for small $Z(F)$ is also evident.

The minimization of the energy functional in Eq. (2) at finite $F$ is equivalent to finding the GS of the Hamiltonian

$$
H(F)=H-F \Delta M(F)
$$

Given the action of $H(F)$ on any diagram, we obtain $\psi(F)$ as usual25 except for one complication. Since we know $Z(0)$ from Eq. (11), but need $Z(F)$ in Eq. (8), iterations are needed. We start with $\Delta M(0)$ to obtain $Z^{(1)}(F)$ and hence $\Delta M^{(1)}(F)$, which is then used in Eq. (9) to find $Z^{(2)}(F)$ and $\Delta M^{(2)}(F)$. We repeat until $Z(F), \psi(F)$ and $P(F)$ have converged. The GS with $\Delta M(0)$ requires the same computational effort as $\psi(0)$ and suffices for the linear response, $\alpha_{e l}=P^{\prime}(0)$. More precisely, we use $\Delta M(0)$ in Eq. (8) to find $\psi(F)$ at small $F$, compute $P(F)$ in Eq. (11) and evaluate $P^{\prime}(0)$. We have $\langle\psi(F)|\Delta M(0)| \psi(F)\rangle=F P^{\prime}(0)$ and, as seen directly from Eq. (7), $\langle\psi(F)|\Delta M(F)| \psi(F)\rangle=0$ The interpretation of Eq. (8) as the induced-dipole operator is now clear: $\Delta M(0)$ generates the linear correction to $P(F)$.

We note that the Berry-phase formulation of $P(F)$ in Eq. (2) differs from standard expressions for finite systems with open boundary conditions. In finite systems, the interaction with a field is $H-F M$ instead of the PBC Hamiltonian in Eq. (9). The dipole operator $M$ is diagonal in the VB basis, with $M_{k}=\sum_{p} p q_{p k}$ for diagram $|k, q\rangle$. Now $\Phi(F)$ is the GS of $H-F M$ and the electronic polarization is $\langle\Phi(F)|M| \Phi(F)\rangle$. In contrast to $\mathrm{PBC}$, there is no iteration and $M_{k}$ is not modulo $N$. The theoretical ideas and computational difficulties that motivated the PBC formulation of $P$ in Eq. (11) and its coupling to $F$ in Eq. (2) do not arise in quantum cell models, whose limited basis is unphysical in this respect. But extrapolations of linear and nonlinear optical coefficients to infinite systems have been widely discussed ${ }^{26}$ for both noninteracting (Hückel) and correlated (Hubbard, Pariser-Parr-Pople) models, especially in connection with frequency-dependent responses. The velocity operator version of $M$ is often used to estimate transition dipole moments for extended (PBC) systems and is consistent with the real-space results $\stackrel{27}{2}$ Susceptibilities obtained with the familiar sum-over-states (SOS) approach require all excited states, a difficult task for systems with large correlated basis, but can be related to GS responses with correction vectors ${ }^{21}$ The calculation of $P(F)$ and its derivatives only requires the GS.

\section{ELECTRONIC POLARIZABILITY OF MODIFIED HUBBARD MODELS}

To illustrate the PBC polarizability of rigid lattices, we consider Hubbard models that have been applied to the electronic structure of both organic CT salts ${ }^{28,29}$ and transition-metal oxides 30 . Face-to-face $\pi$-overlap in CT salts leads to transfer integrals $t$ between sites with energy $-\Delta$ at D and $\Delta$ at $\mathrm{A}$. The modified Hubbard model is 28

$$
\begin{aligned}
H_{H u}= & -\sum_{p, \sigma}\left[1-\delta(-1)^{p}\right]\left(a_{p, \sigma}^{\dagger} a_{p+1, \sigma}+H . c .\right) \\
& +\sum_{p}\left[\Delta(-1)^{p} n_{p}+U \frac{n_{p}\left(n_{p}-1\right)}{2}\right]
\end{aligned}
$$

We take $t=1$ as the unit of energy, consider alternation $0<\delta<1$ along the chain, and assume equal on-site $U>0$ for $\mathrm{A}$ and $\mathrm{D}$ sites. Coulomb or other interactions can be added to $H_{H u}$ without increasing the correlated basis. Such models conserve $S$, have $C_{N / 2}$ symmetry and require comparable effort to obtain the exact GS.

On physical grounds, high-energy $\mathrm{D}^{2+}$ and $\mathrm{A}^{2-}$ sites can be neglected to obtain a restricted basis that is usefully smaller. Formally, we define $\Gamma=\Delta-U / 2$ and take the limit $\Delta, U \rightarrow \infty$ in Eq. (10) while keeping finite $\Gamma$. The electronic problem for rigid lattices simplifies to

$$
\begin{aligned}
H_{0}(\delta, \Gamma)= & -\sum_{p, \sigma}\left[1-\delta(-1)^{p}\right]\left(a_{p, \sigma}^{\dagger} a_{p+1, \sigma}+H . c .\right) \\
& +\sum_{p} \Gamma(-1)^{p} n_{p}
\end{aligned}
$$

The GS for $\Gamma>>0$ is the neutral $(\rho \sim 0)$ lattice with $N$ electrons paired on $N / 2$ donors, while the GS for $\Gamma<<0$ is the ionic lattice $(\rho \sim 1)$ with one electron per site and overall singlet pairing. The regular $(\delta=0)$ stack has an NIT as a function of $\Delta$ in Eq. (10) or $\Gamma$ in Eq. (11) that has been studied by different methods, ${ }^{29}$ including exact GS energies and $\rho$ up to $N=16$ for Eq. (10) and up to $N=22$ for Eq. (111).

In the present work, we take $\Psi(0)$ in Eq. (6) to be the exact GS of $H_{0}(\delta, \Gamma) . P(0)$ in Eq. (1) is the relevant GS dipole per site in units of ea. Then, using $\Delta M(0)$ in Eq. (8), we obtain $\Psi(F)$ exactly, compute $Z(F)$ and $P(F)$, and evaluate $\alpha_{e l}=P^{\prime}(0)$ numerically. Figure 1 , and b show, respectively, $\alpha_{e l}$ and $Z(F)$ for $\delta=0$ stacks of $N=14$ (circles) and 16 (crosses). To understand these 


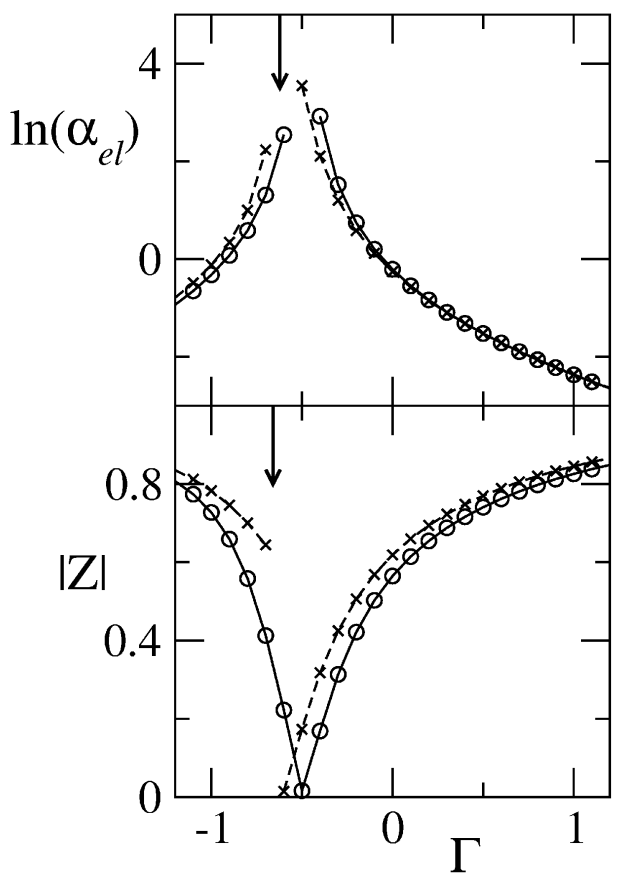

FIG. 1: The logarithm of the linear electronic susceptibility, $\alpha_{e l}$, and the modulus of $Z$ vs $\Gamma$ calculated for the Hamiltonian in Eq. (11) with $\delta=0$ for stacks of 14 and 16 sites (circles and crosses, respectively). The arrows mark the NIT of the infinite strand.

results, we note that regular stacks have real $Z(0)$ by symmetry and hence $P(0)=0$ for any $N$. We have $Z_{x}(0) \sim 1$ at large positive $\Gamma$ where neutral diagrams with $q \sim 0$ dominate in Eq. (5) and $Z_{x}(0) \sim-1$ at large negative $\Gamma$ where ionic diagrams with $q \sim \pm \pi$ dominate. $Z_{x}(0)$ vanishes at some $\Gamma \sim 0$, as seen for both $N=14$ and 16 . The Jahn-Teller instability of $4 n$ rings appears as a symmetry change of $\Psi(0)^{29.31}$ where $Z(0)$ vanishes and changes discontinuously in Fig. 10. The arrow at $\Gamma_{c}=-0.666$ marks the NIT of the extended regular stack as found from extrapolations of symmetry crossovers ${ }^{29}$ Finite-size effects in $Z(0)$ are reduced considerably in $-N \ln \left(|Z(0)|^{2}\right)$, which is the proper sizeindependent quantity 14 The $\alpha_{e l}$ results in Fig. 11 nearly coincide for $N=14$ and 16 except around $Z \sim 0$. In Section 5 we report almost identical $\alpha_{e l}$ for $N=14$ and 16 over the entire $\Gamma$ range for stacks with equilibrium dimerization and $Z \neq 0$.

Figure 2 $\operatorname{shows} \alpha_{e l}(\delta, \Gamma)$ for $\mathrm{N}=16$. Finite alternation, $\delta>0$, strongly suppresses the divergence at the NIT, as seen on comparing $\delta=0.02$ and 0 (dashed line), and shifts the peak to $\Gamma=0$ at $\delta=1$. Regular stacks with a finite potential against dimerization have finite polarizability for $\Gamma \geq \Gamma_{P}>\Gamma_{c}$. The polarizability of dimerized stacks with $\Gamma<\Gamma_{P}$ remains finite because $\delta>0$ opens a gap in the energy spectrum. The $\delta=0.99$ curve follows closely the simple analytical result for $\delta=1$ and

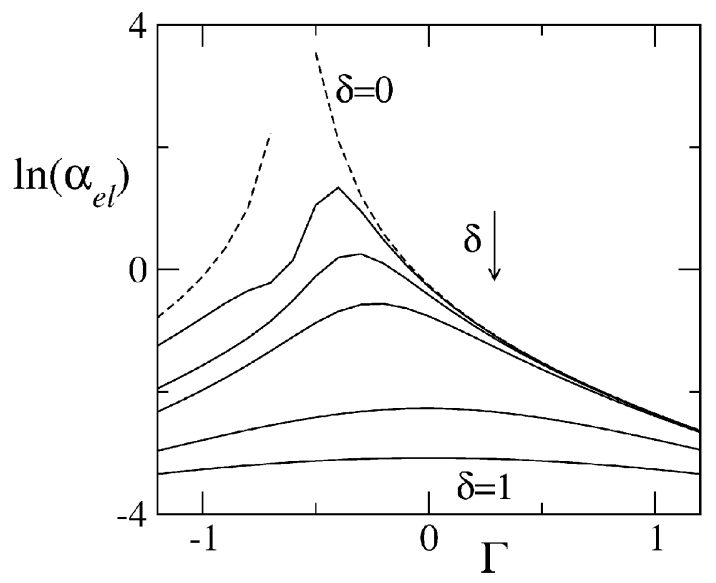

FIG. 2: Logarithm of the linear electronic susceptibility, $\alpha_{e l}$, vs $\Gamma$ for a 16 site stack with $\delta=0.02,0.05,0.1,0.4$ and 0.99 . The arrows mark the direction of increasing $\delta$, and the dashed line for $\delta==0$ diverges at the NIT.

independent dimers,

$$
\alpha_{e l}=\left(\Gamma^{2}+8\right)^{-\frac{3}{2}}
$$

when there is no electronic delocalization. The large variation of $\alpha_{e l}(\delta, \Gamma)$ in Fig. 2 is understood in terms of localization around $\Gamma \sim 0$ due to the dimerization gap and for $|\Gamma|>>0$ due to charge localization for any $\delta$. The pronounced asymmetry of $\alpha_{e l}(\delta, \Gamma)$ with respect to $\Gamma=0$ is due to strong correlations in $H_{0}(\delta, \Gamma)$ leading to quite different GS on the neutral and ionic sides.

We illustrate in Fig. 3 the iterative solution of Eq. (9). Circles refer to the first iteration with $\Delta M(0)$ for $\mathrm{N}=16$ and $\delta=0.1$. Crosses and squares refer to the second and third iteration, respectively. The results for $\alpha_{e l}=P^{\prime}(0)$ do not change, since the linear perturbation in $F$ appears from the outset and iterations introduce higher-order corrections. Finite $\delta$ is required for finite $P^{\prime \prime}(0)$ and $F^{2}$ corrections must be included. The second iteration more than doubles $P^{\prime \prime}(0)$, but the third iteration produces no additional change. Taylor expansion of $\Delta M(F)$ through the linear term suffices for $F^{2}$ contributions. Nonlinear polarizabilities based on the PBC formulation in Eq. (2) or (9) raise interesting issues for rigid lattices. Here we focus instead on vibronic contributions in Eq. (3) to the linear polarizability of models with Peierls transitions.

\section{POLARIZABILITY NEAR THE PEIERLS TRANSITION}

The Su-Schrieffer-Heeger (SSH) mode ${ }^{32}$ for polyacetylene, $(\mathrm{CH})_{x}$, describes in the adiabatic approximation the Peierls instability of Hückel chains with $U=\Delta=0$ in Eq. (10) and linear e-ph coupling $\alpha_{e-p h}=t \delta / u$. Similar approaches have been applied to Peierls transitions in segregated $(\Delta=0)$ stacks of $\pi$-electron donors or 


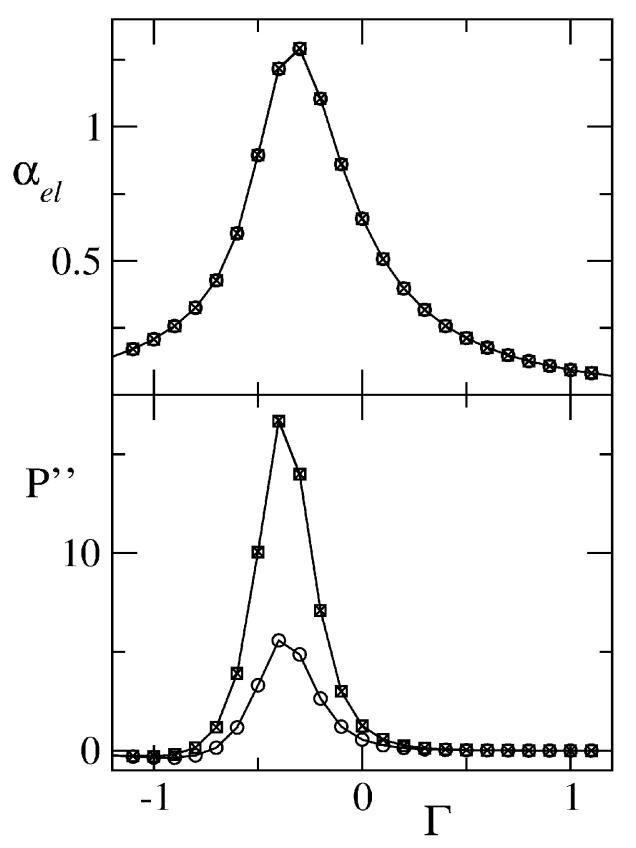

FIG. 3: The linear electronic susceptibility, $\alpha_{e l}$, and the second derivative of the GS polarization on the electric field, $P^{\prime \prime}$, calculated as a function of $\Gamma$ for a 16 site stack with $\delta=0.1$. Circle, squares and crosses refer to results obtained at the first second and third iteration, respectively (see text).

acceptors, 33 or to uncorrelated $(U=0)$ chains ${ }^{34}$ with site energies $\pm \Delta$. The stability of mixed stacks described by $H_{0}$ has been studied through the response of a regular lattice to the SSH coupling, 31 In the adiabatic approximation, the GS potential energy surface is defined by adding the elastic energy for lattice motion to the electronic energy. For harmonic potentials and linear electron-phonon coupling, the elastic energy of the Peierls mode is $\delta^{2} / 2 \epsilon_{d}$ per site, where $\epsilon_{d}=\alpha_{e-p h}^{2} / k$ is the small polaron binding energy and $k$ is the lattice force constant ${ }^{22}$ We therefore define the Peierls-Hubbard model by adding the lattice energy to $H_{0}(\delta, \Gamma)$ in Eq. (11). The electronic energy in an applied field is given by Eq. (2). The total GS energy per site is

$$
\mathcal{E}_{T}=\mathcal{E}_{0}(\delta, \Gamma, F)+\frac{\delta^{2}}{2 \epsilon_{d}}
$$

The same development holds for arbitrary $U, \Delta$ in Eq. (10) or for other cell models with PBC.

Eq. (13) fully defines the GS potential energy surface with one additional parameter, the lattice stiffness $1 / \epsilon_{d}$, besides those entering the electronic Hamiltonian. The equilibrium dimerization is obtained by minimizing of the total energy,

$$
\delta_{e q}=-\epsilon_{d} \frac{\partial \mathcal{E}_{0}(\delta, \Gamma, F)}{\partial \delta}
$$

The limiting inverse stiffness is $\epsilon_{d}=\sqrt{2}$, which yields an equilibrium dimerization in Eq. (14) of $\delta=1$ at $\Gamma=0$

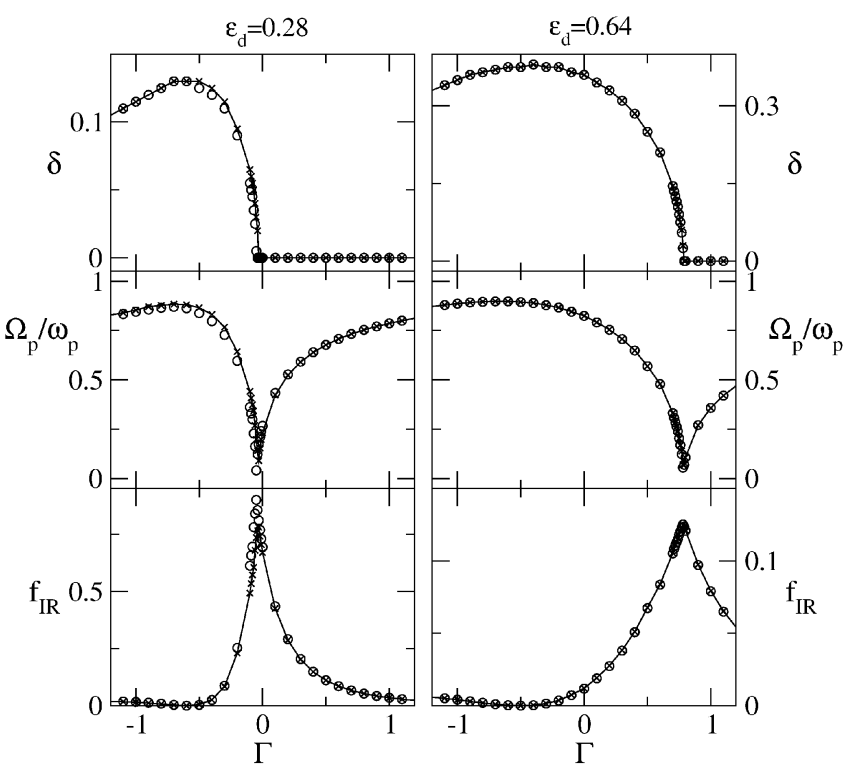

FIG. 4: Equilibrium dimerization, $\delta$, softened frequency $\Omega_{P}$ in units of the reference frequency $\omega_{P}$, and infrared oscillator strength, calculated for Eq. (13) with $\epsilon_{d}=0.28$ and 0.64 (left and right panels, respectively.) Circles refer to $N=14$, crosses to $N=16$.

for a stack of decoupled dimers whose GS is easily found. The top panel of Fig. 4 shows the equilibrium dimerization of $H_{0}(\delta, \Gamma)$ at $F=0$ for $\epsilon_{d}=0.28$ and 0.64 in units of $t$. Since results for $N=14$ (crosses) and 16 (circles) now coincide almost exactly, we conclude that finite-size effects have become negligible in deformable lattices with $\delta>0.1$ around $\Gamma \sim 0$ that precludes strong delocalization.

Previous modeling of TTF-CA used the estimate $\epsilon_{d} \sim$ $0.15-0.33^{31.35}$ The $\epsilon_{d}=0.64$ stack is less stiff, with a three-fold increase in the maximum dimerization to $\delta_{e q} \sim 0.3$ around $\Gamma \sim 0$. The Peierls instability occurs at $\left(\partial^{2} \mathcal{E}_{\mathcal{T}} / \partial \delta^{2}\right)_{0}=0$, where the curvature $1 / \epsilon_{d}$ of the lattice potential and the curvature of the electronic energy cancel exactly. The stack is regular for $\Gamma>\Gamma_{P}\left(\epsilon_{d}\right)$ and dimerized for $\Gamma<\Gamma_{P}\left(\epsilon_{d}\right)$. As expected on general grounds, the neutral lattice with $\Gamma>>0$ is a band insulator that is conditionally stable. The divergence of $\left(\partial^{2} \mathcal{E}_{0} / \partial \delta^{2}\right)_{0}$ at $\Gamma_{c}$ ensures a Peierls instability at some $\Gamma_{P}>\Gamma_{c}$ that is model dependent. Dimerization decreases but persists as $\Gamma$ becomes more negative ${ }^{31}$ The $\Gamma<<0$ limit with $\rho \sim 1$ maps into the spin- $1 / 2$ Heisenberg antiferromagnetic chain ${ }^{36}$ whose GS is unstable to a spin-Peierls transition ${ }^{\frac{5}{}}$

The curvature of the GS potential energy surface is the frequency of the Peierls mode $\Omega_{P}^{2}=\left(\partial^{2} \mathcal{E}_{\mathcal{T}} / \partial \delta^{2}\right)_{e q}$. At $F=0$ the ratio $\Omega_{P} / \omega_{P}$ at the equilibrium $\delta$ is a function of $\Gamma$ :

$$
\left(\frac{\Omega_{P}}{\omega_{P}}\right)^{2}=1+\epsilon_{d}\left(\frac{\partial^{2} \mathcal{E}_{0}(\delta, \Gamma)}{\partial \delta^{2}}\right)_{e q}
$$


The middle panel of Fig. [4shows the evolution of $\Omega_{P} / \omega_{P}$ for the correlated model $H_{0}(\delta, \Gamma)$ in Eq. (II). The softening of the Peierls mode on the neutral side and its subsequent hardening are observed for $\mathrm{TTF}-\mathrm{QBrCl}{ }_{3}$ whose continuous dimerization transition has been investigated by far-IR spectroscopy 10 The temperature evolution of a combination band in the mid-IR spectrum of TTF-CA for $T>T_{c}$, also supports the presence of a soft lattice mode $\stackrel{37}{2}$ The data are in both cases consistent with $\omega_{P} \sim$ $100 \mathrm{~cm}^{-1}$, a typical frequency for lattice vibrations in crystals with molecular masses of $\sim 100$ AMU.

The Peierls mode is IR active by symmetry in mixed stacks and borrows huge IR intensity from electronic degrees of freedom ${ }^{22}$ In the present approximation, with $M$ in Eq. (4) for fixed sites, the IR intensity of the Peierls mode is entirely due to charge fluctuations induced by $\delta$. The corresponding oscillator strength is:

$$
f_{I R}=\frac{2 m_{e} \Omega_{P}\left|\mu_{I R}\right|^{2}}{e^{2} \hbar}=\frac{m_{e} a^{2} \omega_{P}^{2} \epsilon_{d}}{t}\left(\frac{\partial P}{\partial \delta}\right)_{e q}^{2}
$$

Here $m_{e}$ and $e$ are the electronic mass and charge, respectively, $\left|\mu_{I R}\right|^{2}$ is the squared transition dipole per site, and $\epsilon_{d}$ is in units of $t$. Once again the IR intensity is governed by $P$-derivatives and $\epsilon_{d}$. However $\omega_{P}, a$ and $t$ enter the expression for the dimensionless $f_{I R}$. The bottom panels of Fig. 团 show $f_{I R}$ for typical parameters $\stackrel{35}{=} a=3.7 \AA$, $\omega_{P}=100 \mathrm{~cm}^{-1}, t=0.2 \mathrm{eV}$.

The intensity of the Peierls mode has a pronounced peak at $\Gamma_{P}$ where the electronic charges are maximally mobile 22 Dimerization localizes charges and lowers $f_{I R}$ for $\Gamma<\Gamma_{P}$. We have $f_{I R}=0$ when the electronic flux induced by dimerization reverses from $\mathrm{D} \rightarrow \mathrm{A}$ on the neutral side to $\mathrm{A} \rightarrow \mathrm{D}$ on the ionic side. The neutralionic interface of deformable lattices can be identified as $f_{I R}=0$, which occurs near $\Gamma=\Gamma_{c}$ for regular stacks or $Z_{x}(\delta, \Gamma)=0$ in dimerized stacks ${ }^{22}$ The actual IR intensity does not vanish due to the motion of the molecular sites with charges $\pm e \rho$, but frozen charge contributions ${ }^{22}$ are neglected in Eq. (4). In materials that dimerize in the neutral side, the N-I interface could, in principle, appear experimentally as a dip in $f_{I R}$. However, this dip does not mark a phase transition. There is no change in symmetry and all properties of the system vary continuously at the interface 31.38

The IR intensity of the stiff $\left(\epsilon_{d}=0.28\right)$ stack in Fig. 4 is an order of magnitude larger than that of the $\epsilon_{d}=$ 0.64 stack. While contrary to the $\epsilon_{d}$ factor in Eq. (16), this is readily understood in terms of greater delocalization in stiff lattices whose $\Gamma_{P}$ approaches $\Gamma_{c}$ and whose $\partial P / \partial \delta$ diverges in the limit $\epsilon_{d} \rightarrow 0$. At the Peierls transition, the large oscillator strength of the dimerization mode corresponds to effective masses $m^{*}=m_{e} / f_{I R} \sim$ 1000 and 6000 for $\epsilon_{d}=0.28$ and 0.64 , respectively. So $m^{*}$ at the transition is roughly a proton mass and is fully two orders of magnitude smaller than molecular masses. This justifies a posteriori the neglect of frozen charge contributions to $f_{I R}$ near the Peierls transition. ${ }^{22}$

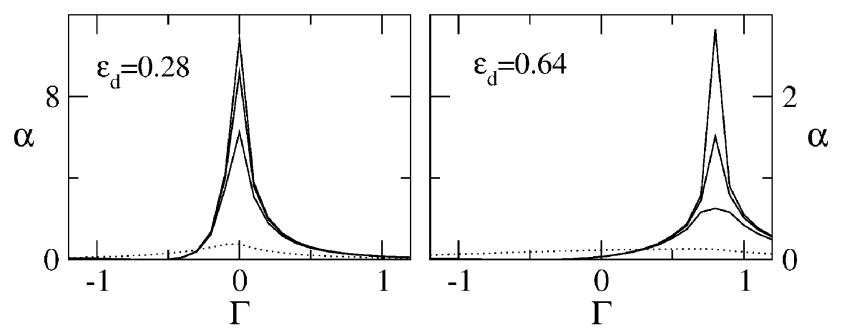

FIG. 5: Electronic and vibrational contributions to the static polarizability (dotted and continuous lines, respectively), for $N=16$ and the same parameters as in Fig. 4 The three lines for $\alpha_{v i b}$ have, from top to bottom, for $\gamma / \omega_{P}=0.01,0.1$, 0.2 .

The small effective mass associated with dimerization at the Peierls transition implies strong mixing of electronic and vibrational degrees of freedom. Lattice contributions to the polarizability can be substantial, as anticipated for $\alpha_{v i b}$ in Eq. (3). To demonstrate this, we differentiate both sides of Eq. (14) with respect to $F$ and use $P=-\left(\partial \mathcal{E}_{0} / \partial F\right)$ to obtain

$$
\frac{\partial \delta_{e q}}{\partial F}=\frac{\epsilon_{d}}{1+\epsilon_{d}\left(\partial^{2} \mathcal{E}_{0} / \partial \delta^{2}\right)_{e q}} \frac{\partial P}{\partial \delta}
$$

We then use Eq. (15) to write the vibrational polarizability of the Peierls mode at $\delta_{e q}$ :

$$
\alpha_{v i b}=\epsilon_{d}\left(\frac{\omega_{P}}{\Omega_{P}}\right)^{2}\left(\frac{\partial P}{\partial \delta}\right)^{2} \rightarrow \frac{\epsilon_{d} \omega_{P}^{2}}{\Omega_{P}^{2}+\gamma^{2}}\left(\frac{\partial P}{\partial \delta}\right)^{2}
$$

This expression is exact when the vibrational kinetic energy is neglected, which is a good approximation in view of the low frequencies involved ${ }^{39}$ It is equivalent to the sum-over-states expression, ${ }^{39} \alpha_{v i b}=2\left|\mu_{I R}\right|^{2} / \hbar \Omega_{P}$, based on IR transition moments and energies.

Large $\alpha_{v i b}$ is expected for the dimerization mode due to its large transition dipole and low frequency. Indeed, $\Omega_{P}=0$ at the Peierls transition gives a divergent polarizability. We suppress the divergence by introducing damping $\gamma$ that represents the lifetimes of lattice modes, anharmonic potentials, etc. Damping is introduced empirically by changing $\left(\Omega_{P} / \omega_{P}\right)^{2}$ as shown in the second equality in Eq. (18). Figure 5 reports $\alpha_{v i b}$ for the $\epsilon_{d}=$

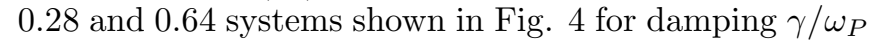
$=0.01,0.1$ and 0.2 . The dotted line is the electronic polarizability $P^{\prime}(0)$ of the deformable lattice. The indicated damping corresponds to $\gamma=1,10$ and $20 \mathrm{~cm}^{-1}$ for an estimated $\omega_{P}=100 \mathrm{~cm}^{-1}$. Quite predictably, damping affects $\alpha_{v i b}$ in a narrow region about $\Gamma_{P}$ and is more pronounced in the softer lattice. We note that typical bandwidths of lattice modes in molecular crystals are in the $1-10 \mathrm{~cm}^{-1}$ range. We will use $\gamma=0.1 \omega_{P}$ to model the dielectric response of $\mathrm{CT}$ salts and cannot specify $\alpha_{v i b}$ peaks more accurately than shown in Fig. [5] 


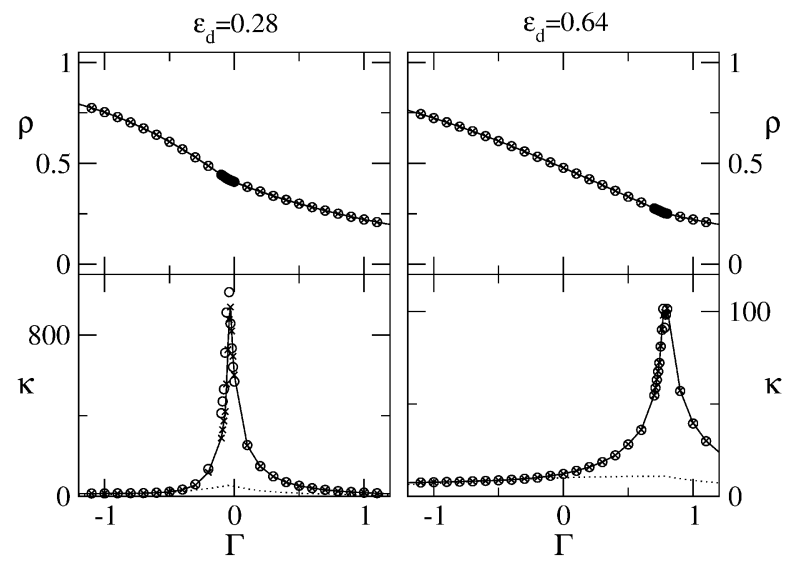

FIG. 6: The ionicity, $\rho$, and the dielectric response, $\kappa$, calculated for the same parameters as in Fig. 4 Circles refer to $\mathrm{N}=14$, crosses to $\mathrm{N}=16$. Dotted lines in the bottom panels show the dielectric response obtained by neglecting the vibrational contribution to $\alpha$.

\section{DIELECTRIC ANOMALY OF PEIERLS-HUBBARD MODELS}

We now combine the electronic and vibrational polarizabilities of the Peierls-Hubbard model in Eq. (11) to obtain the dielectric constant of the equilibrium lattice. In SI units,

$$
\kappa=\kappa_{\infty}+\frac{\alpha_{e l}+\alpha_{v i b}}{\epsilon_{0} v}
$$

Here $\epsilon_{0}$ is the vacuum permittivity constant, $\kappa_{\infty} \sim 3$ is the usual contribution from molecular excited states that are not being modeled, and $v$ is the volume per site. We are interested in remarkably large peaks with $\kappa>100$. To get proper dimensions for $\kappa$ the polarizabilities $\left(\alpha_{e l}\right.$ and $\left.\alpha_{v i b}\right)$ are multiplied by $e^{2} a^{2} / t$. We adopt typical TTF-CA values, $v=206 \AA^{3}, a=3.7 \AA$, and $t=0.2 \mathrm{eV}$, and in the lower panels of Fig. [6] we show the resulting $\kappa=3+60\left(\alpha_{e l}+\alpha_{v i b}\right)$ for $N=14$ and 16 stacks with equilibrium dimerization and $\epsilon_{d}=0.28$ and 0.64 . The dotted lines are the electronic contribution to $\kappa$. The dielectric peak is clearly associated with the Peierls transition and vibrations. The divergence of $\alpha_{e l}$ in Fig. 1 at the NIT $\left(\Gamma_{c}=-0.666\right)$ of the $\delta=0$ stack is strongly attenuated by dimerization and appears as a small bump around $\Gamma_{P}$ in Fig. 6] The electronic fluxes leading to the IR intensity of the Peierls mode are also responsible for the large $\kappa$ peaks of $\sim 1000$ and 100 estimated for $\epsilon_{d}=0.28$ and 0.64 , respectively. We note that neglecting the softening, i.e. by imposing $\Omega_{P}=\omega_{P}$ in Eq. (18), lowers the $\kappa$ peak by an order of magnitude, while neglecting damping $(\gamma=0)$ gives a divergence. We took $\gamma=\omega_{P} / 10=10 \mathrm{~cm}^{-1}$ in Fig. 6 .

Both the height and shape of the $\kappa$ peaks as a function of temperature compare favorably to the dielectric

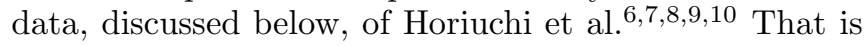

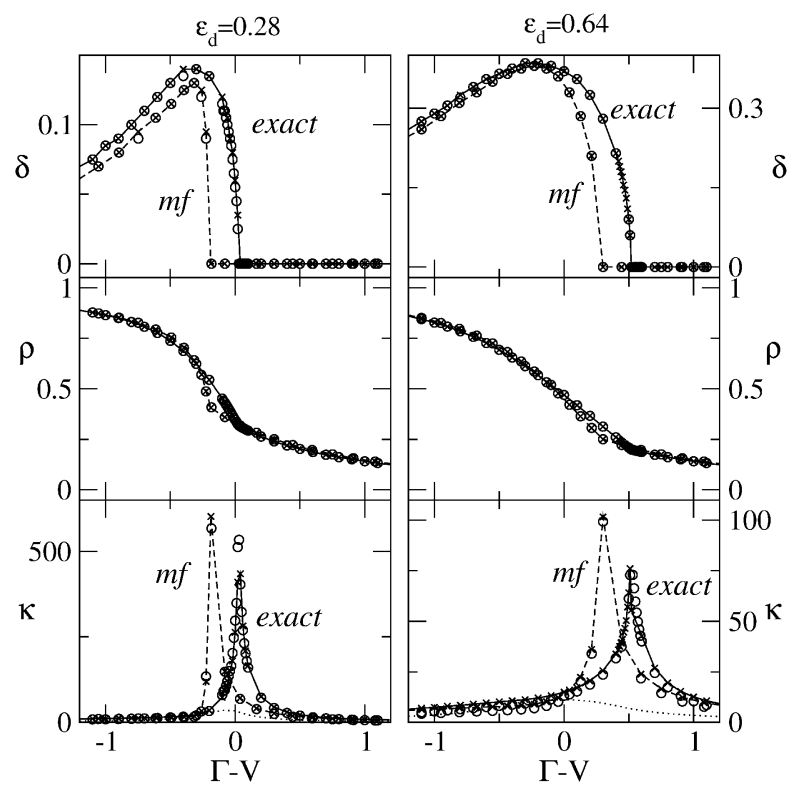

FIG. 7: The equilibrium dimerization amplitude, $\delta$, the GS ionicity, $\rho$, and the dielectric response, $\kappa$, for the same parameters as in Fig. 4 except for $V=2$. Circles and crosses refer to $N=14$ and 16, respectively, with continuous and dashed lines joining exact and $\mathrm{mf}$ results. Dotted lines in the bottom panels are the electronic mf dielectric response for $N=16$.

not the case for the ionicity $\rho$ shown in the upper panel of Fig. [6. We properly have $\rho \sim 0.40$ at the Peierls transition of the $\epsilon_{d}=0.28$ stack and $\rho \sim 0.25$ in the softer stack, but $\rho(\Gamma)$ hardly shows any sign of a transition. By contrast, the measured ionicity has a kink in systems with continuous transitions around $\rho \sim 0.25 \stackrel{6.7 .8 .9 .10}{=}$ The basic model $H_{0}(\delta, \Gamma)$ in Eq. (11) is deficient in this respect, just as its continuous NIT in rigid lattices cannot account for discontinuous $\rho$ at $T_{c} \sim 81 \mathrm{~K}$ in TTF$\mathrm{CA}$. Several extensions of the model have long been recognized. Coulomb interactions occur both within and between stacks. ${ }^{28.40}$ Coupling to molecular modes is another general phenomenon ${ }^{31}$ In practice, mean-field (mf) approximations are necessary for inter-stack interactions. While observing a $\rho$ anomaly at the Peierls transition requires going beyond $H_{0}(\delta, \Gamma)$, the appropriate extensions and parameters remain open.

To illustrate a simple extension that is suitable for either exact or mf analysis, we introduce nearest-neighbor Coulomb interactions $V$ along the stack,

$$
H_{V}=H_{0}(\delta, \Gamma)+V \sum_{p} q_{p} q_{p+1}+N \frac{\delta^{2}}{2 \epsilon_{d}}
$$

Since $V$ is diagonal in the VB basis, all GS properties are found as before. The solid lines in Fig. 7 are exact $N=14$ and 16 results for the equilibrium dimerization, ionicity and dielectric anomaly of stacks with $V=2$ and otherwise the same parameters as in Figs. 4 and 6 The dashed lines are the $\mathrm{mf}$ approximation to the $V$ term in 


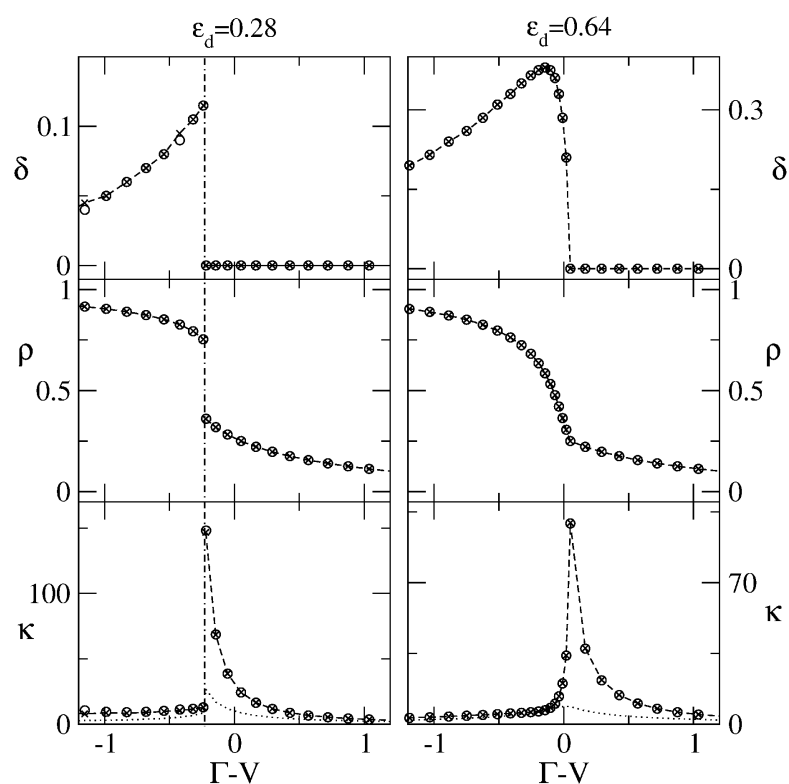

FIG. 8: The same as Fig. 7 for $V=3$; for the sake of clarity only $\mathrm{mf}$ results are reported, with circles and crosses referring to $N=14$, and 16, respectively. Dotted lines in the lowest panels show the electronic contribution to $\kappa$.

Eq. (20). The mf results for $V=2$ are similar, with somewhat sharper $\rho$ at $\Gamma_{P}$ as expected for an interaction that stabilizes adjacent ion-pairs. Both $\epsilon_{d}=0.28$ and 0.64 now have a kink at $\rho\left(\Gamma_{P}\right)$ that qualitatively resembles $\rho(T)$ data. The $\kappa$ peak is lower by a factor of two, while the maximum dimerization hardly changes. Charges are kept together for $V>0$, thereby reducing the polarizability.

The first-order transition of TTF-CA at $81 \mathrm{~K}$ has a $\Delta \rho \sim 0.2$ and concomitant neutral-ionic and Peierls transitions $\stackrel{2.3 .4}{2}$ The ionicity is a suitable order parameter in this case. Discontinuous $\rho$ has long been treated in $\mathrm{mf}$ theory ${ }_{2}^{28}$ and $\mathrm{mf}$ results for $V=3$ are shown in Fig. 8 for the same quantities and parameters as in Fig. 7 Now $\epsilon_{d}=0.28$ produces a $\Delta \rho$ jump in stacks of $N=$ 14 and 16 that resemble previous results for smaller $N$

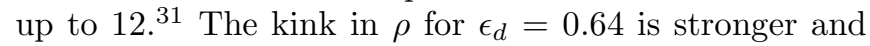
still larger $V$ will make it discontinuous. The maximum dimerization remains almost independent of $V$. The $\kappa$ peak now appears at the NIT and is reduced for both $\epsilon_{d}=0.28$ and 0.64 . The reason for reduced vibrational contributions is that a discontinuous NIT interrupts the softening of $\omega_{P}$. This is shown in Fig. 9 for the same parameters, together with $f_{I R}$.

Figure 7 contrasts exact and $\mathrm{mf}$ results for small $\mathrm{V}=$ 2, while Fig. 8 shows a first-order $\mathrm{mf}$ transition for $\mathrm{V}=$ 3 in the stiff stack. To summarize the various roles of $V$ in Eq. (20), we note that in $\mathrm{mf}$ it also accounts for the coupling of electrons with molecular vibrations. Totallysymmetric (ts) molecular vibrations modulate on-site energies, and hence $\Gamma$, leading to Holstein coupling. $\underline{\underline{41}}$ The

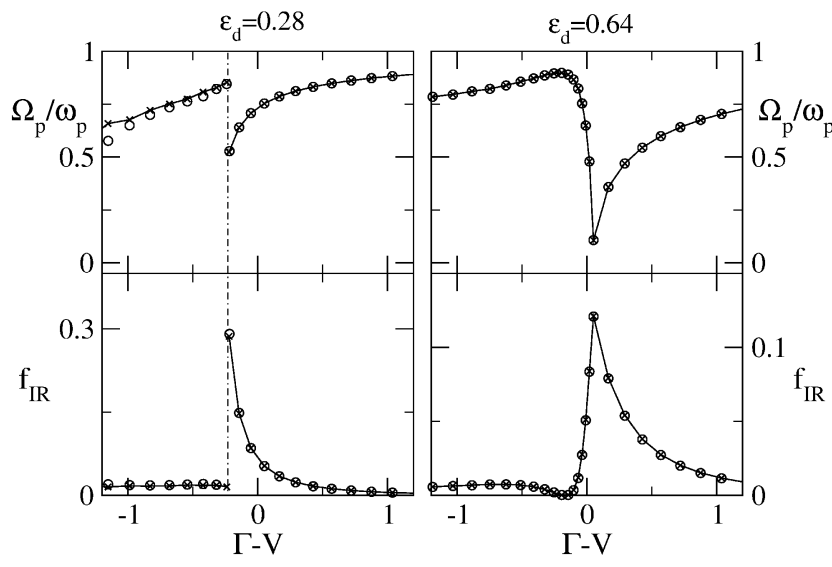

FIG. 9: Mean-field results for the softening of the Peierls mode and its IR intensity, calculated for the same parameters as in Fig. 8 Circles refer to $N=14$, crosses to $N=16$.

effects of this coupling on vibrational spectra of CT salts are well known ${ }^{42.43 .44}$ In regular stacks, ts vibrations only appear in Raman spectra, whereas in dimerized stacks they acquire large IR intensity, proportional to $(\partial P / \partial \Gamma)^{2}$, through their coupling to electronic degrees of freedom ${ }^{45}$ In the adiabatic approximation, Holstein coupling plays exactly the same role as $V$ within $\mathrm{mf} \mathrm{si}^{31.45}$ Thus mf results in Figs. [7] and 8/represent an effective parameter $V$ whose interpretation is model dependent. Although the large IR intensity of Holstein modes in dimerized lattices suggests that also they contribute to $\kappa$, their high frequency $\left(\sim 1000 \mathrm{~cm}^{-1}\right)$ makes this contribution negligible in comparison to the Peierls modes.

\section{DISCUSSION}

The dipole $P$ for insulators with $\mathrm{PBC}$ and its extension in Eq. (2) to an applied field, make it possible to model the electronic and vibrational polarizability of quantum cell models. We found the induced-dipole operator $\Delta M$ in Section 2 for quantum cell models in general and applied it to a modified Hubbard model, $H_{0}(\delta, \Gamma)$ in Eq. (111). We report model-exact electronic polarizabilities in Fig. 2 for the NIT of regular $(\delta=0)$ and dimerized stacks. We then include the Peierls mode with frequency $\omega_{P}$ in the adiabatic approximation and its softening. The IR intensity of the Peierls mode in Fig. 4 and the dielectric peaks in Figs. 6 and 7 are related to Peierls transitions in stacks with stiffness $1 / \epsilon_{d}$. We have chosen $\epsilon_{d}=0.28$ as a plausible estimate for TTF-CA and a softer stack with $\epsilon_{d}=0.64$ whose Peierls transition is around $\rho \sim 0.3$ on the neutral side. The choice of $t=$ $0.2 \mathrm{eV}$ comes from TTF-CA optical data. The model parameters are not arbitrary, but typical of values used for other properties ${ }^{31.35}$ The same analysis holds for various extensions of $H_{0}(\delta, \Gamma)$ for organic CT salts. All results are derived from the $\Gamma$-dependence of the GS properties 
of stacks described by $H_{0}(\delta, \Gamma)$, and at the equilibrium dimerization for fixed $\epsilon_{d}$ in Eq. (13) and fixed nearestneighbor interactions $V$ in Eq. (20).

The dielectric data of Horiuchi et al. are taken as a function of temperature at ambient pressure, $\stackrel{6.7 .8 .9 .10}{\sim}$ or, more recently, as a function of pressure, up to $\sim 10 \mathrm{kbar}$, at constant temperature 11 Decreasing volume on cooling or compression stabilizes the ionic phase by increasing the crystal's Madelung energy. Cooling corresponds to decreasing $\Gamma$ and increasing $\rho$ in models, but the relation between temperature or pressure and $\Gamma$ is not known at present. Cooling also increases DA overlaps that enter in $t$ and $\epsilon_{d}$ for one-dimensional models. Overlap between stacks also increases, but although the importance of inter-stack interactions has been noted,,$\frac{4}{\underline{\underline{n}}}$ their modeling is still rather qualitative.

The crystals studied by Horiuchi et al. in refs. 9, 11 have $\mathrm{D}=\mathrm{DMTTF}$ (dimethyl-TTF) and the quinones $\mathrm{A}$ $=\mathrm{QBr}_{n} \mathrm{Cl}_{4-n}$ with $n=0-4 ; n=0$ and 4 are CA and bromanil (BA), respectively. The acceptors have twofold disorder in $\mathrm{Br} / \mathrm{Cl}$ in the crystal lattice for $n=1,2$ and 3. There are two $\mathrm{QBr}_{2} \mathrm{Cl}_{2}$ isomers, a centrosymmetric one called 2,5 and a polar one called 2,6. The $n=1,3$ and 2,6 complexes are formally polar, but only weakly so: Their dielectric peaks are similar but are shifted to lower temperature. As there is no disorder in the prototypical system, TTF-CA, the best candidates with continuous transitions are DMTTF-CA and DMTTF-2, $5 \mathrm{QBr}_{2} \mathrm{Cl}_{2}$. The dielectric peak of DMTTF-CA at $65 \mathrm{~K}$ is $\kappa \sim 220$ at $30 \mathrm{kHz} ; \rho$ increases quickly from $\sim 0.32$ to $\sim 0.42$ between 65 and $60 \mathrm{~K}$ and then slowly to $\sim 0.48$ at 10 $\mathrm{K}$. The DMTTF-2,5QBr ${ }_{2} \mathrm{Cl}_{2}$ peak at $50 \mathrm{~K}$ is $\kappa \sim 170$ at $30 \mathrm{kHz}$, with $\rho$ increasing from 0.29 to 0.36 on cooling from 50 to $10 \mathrm{~K}$. The $\kappa$ peak of DMTTF-QBrCl 3 at $\sim 55$ $\mathrm{K}$ closely resembles the 2,5 system, but $\rho$ has not been reported. Pressure-induced $\kappa$ peaks are reported 11 at 5 $\mathrm{K}$ in DMTTF-BA $(n=4)$ and in DMTTF- $2,6 \mathrm{QBr}_{2} \mathrm{Cl}_{2}$, both with $\rho<0.3$ at $5 \mathrm{~K}$ and $1 \mathrm{~atm}$.

The observed peak heights of $\kappa$ are consistent with dielectric peaks of the model at the Peierls transition. We conclude that a $\kappa$ peak marks the Peierls transition in systems with continuous $\rho$. The narrower $\kappa$ peak in Fig. 8 for model parameters leading to a first-order transition is also seen in the narrow $\kappa=600$ peak of TTF-CA $\frac{8}{2}$ The peak shapes and heights follow qualitatively the calculated pattern of increased polarizability in stiff lattices with Peierls transitions (continuous $\rho$ ) occurring at $\Gamma_{P}$ close to $\Gamma_{c}$. More quantitative analysis requires a relation between temperature and $\Gamma$. As illustrated in Section 5 , accurate modeling of $\rho(\Gamma)$ near $\Gamma_{P}$ depends on such specifics as damping and intra and inter-stack interactions. By contrast, the Peierls transition of deformable stacks and NIT of rigid stacks are general features of Hubbard and related models.

The dielectric anomaly observed in mixed stack CT salts is clearly associated with lattice degrees of freedom and the Peierls transition in systems with either continuous or discontinuous NIT. The huge IR intensity of the Peierls mode around the Peierls transition ${ }^{22}$ and its softening are responsible for $\alpha_{v i b}>>\alpha_{e l}$ in Eq. (3). Available spectroscopic data 10 in the far-IR region for TTF-QBrCl ${ }_{3}$ support this picture ${ }^{22}$ It is interesting to compare the Peierls transition occurring in this system at $\rho \sim 0.3$ with the discontinuous NIT occurring in TTFCA at similar $\rho$ : in TTF-CA the NIT occurs before the complete softening of the Peierls mode and a reduced $\kappa$ peak is observed. Whereas far-IR data are not available for TTF-CA, the incipient softening of the dimerization mode has been recently extracted from a detailed study of combination bands in the mid-IR region ${ }^{37} \mathrm{~A}$ more systematic analysis of far-IR spectra and dielectric properties of mixed stack materials is certainly desirable as it will confirm the important connection between lattice degrees of freedom and materials properties.

Organic CT crystals have DA repeat units along the stack and, in contrast to most inorganic salts, are quasione-dimensional systems. Rapid convergence, often as $1 / N^{2}$, is typical for GS properties of systems with PBC and small repeat units. It is very convenient for modeling that $P(F)$ is a GS property. As found throughout, all $N=14$ and $N=16$ results coincide in deformable lattices with $\delta>0.1$ at $\Gamma \sim 0$. Larger $\mathrm{N}$ is not required for CT salts, but is needed for the NIT of $\delta=0$ stacks.

Discontinuous $\rho$ at the NIT is fundamentally different from continuous $\rho$ at the Peierls transition of softer lattices. We have focused on the Peierls-Hubbard model, $H_{0}(\delta, \Gamma)$ in Eq. (11), which has continuous $\rho$ and a Peierls instability on the neutral side for large $\epsilon_{d}$. The order parameter $\delta$ for the second-order transition breaks the reflection symmetry of the regular chain. Once the stack has dimerized, there is no further transition and, indeed, hardly any remnants of the prominent neutral-ionic transition of the $\delta=0$ stack. Models with continuous $\rho$ have Peierls rather than neutral-ionic transitions. Similar results are obtained for stacks with not too large $V$-like interactions: The Peierls or structural transition occurs at small $\rho$, and $V$ promotes a more prominent kink in $\rho$. Increasing further $V$ (or Holstein) interactions leads to a discontinuous NIT, where the on-site charge reorganization is the driving event, $\rho$ is the proper order parameter, and dimerization just follows from the unconditional instability of the ionic phase.

The increasing number of CT salts undergoing phase transitions demonstrates 46 far more diversity than the neutral-regular and ionic-dimerized GS of TTF-CA. The lattice stiffness $1 / \epsilon_{d}$ is an important parameter for distinguishing between Peierls and neutral-ionic transitions. Disordered D or A in single crystals raises different issues. The microscopic modeling of CT salts has primarily been in terms of Hubbard-type models with many open questions about parameters. We anticipate that accurate modeling of $P(F)$ and its applications to dielectric, vibrational and other data will lead to more quantitative descriptions of quantum phase transitions in these materials. 


\section{Acknowledgments}

Z.S. thanks R.Resta for fruitful discussions, and A.P. likewise thanks S. Ramasesha and F. Terenziani. Work in Parma was partly supported by the Italian Ministry of Education (MIUR) through COFIN-2001, and by INSTM through PRISMA-2002.
1 J.B. Torrance, J.E. Vasquez, J.J. Mayerle and V.Y. Lee, Phys. Rev. Lett. 46, 253 (1981); J.B. Torrance, A. Girlando, J.J. Mayerle, J.I. Crowley, V.Y. Lee, P. Batail and S.J. LaPlaca, Phys. Rev. Lett. 47, 1747 (1981).

2 A. Girlando, F. Marzola, C. Pecile and J.B. Torrance, J. Chem. Phys. 79, 1075 (1983).

3 K.Takaoka, Y. Kaneko, H. Okamoto, Y. Tokura, T. Koda, T. Mitani and G. Saito, Phys. Rev. B, 36, 3884 (1987).

4 M. Le Cointe, M.H. Lemee-Cailleau, H. Cailleau, B. Toudic, L. Toupet, G. Heeger, F. Moussa, P. Schweiss, K. H. Kraft and N. Karl, Phys. Rev. B, 51, 3374 (1995).

5 J.W. Bray, L.V. Interrante, I.S. Jacobs and J.C. Bonner, in Extended Linear Chain Compounds, J.S. Miller, ed. (Plenum, New York, 1983), Vol. 3, p. 353.

6 S. Horiuchi, R. Kumai, and Y. Okimoto and Y. Tokura, Phys. Rev. B, 59, 11267 (1999).

7 S. Horiuchi, R. Kumai, Y. Okimoto and Y. Tokura, Phys. Rev. Lett. 85, 5210 (2000).

8 S. Horiuchi, Y. Okimoto, R. Kumai and Y. Tokura, J. Phys. Soc. Japan 69, 1302 (2000).

9 S. Horiuchi, Y. Okimoto, R. Kumai and Y. Tokura, J. Am. Chem. Soc. 123, 665 (2001).

10 Y. Okimoto, S. Horiuchi, E. Saitoh, R. Kumai and Y. Tokura, Phys. Rev. Lett. 87, 187401 (2001).

11 S. Horiuchi, Y. Okimoto, R. Kumai and Y. Tokura, Science 299, 229 (2003).

12 R.D. King-Smith and D. Vanderbilt, Phys. Rev. B 47, 1851 (1993); 48, 4442 (1993); R. Resta, Rev. Mod. Phys. 66, 899 (1994).

13 R. Resta, Phys. Rev. Lett. 80, 1800 (1998).

14 R. Resta, S.Sorella, Phys. Rev. Lett. 82, 370 (1999); R. Resta, J. Phys: Cond. Mat. 14, R625 (2002).

15 A. A. Aligia and G. Ortiz, Phys. Rev. Lett., 82, 2560 (1999); M. Nakamura and J. Voit, Phys. Rev. B 65, 153110 (2002).

16 R.W. Nunes and X. Gonze, Phys. Rev. B 63, 155107 (2001).

17 I. Souza, T. Wilkins and R.M. Martin, Phys. Rev. B 62, 1666 (2000).

18 P. Umari and A. Pasquarello, Phys. Rev. Lett. 89, 157602 (2002).

19 I. Souza, J. Iniguez and D. Vanderbilt, Phys. Rev. Lett. 89, 117602 (2002).

20 G.M. Rignanese, F. Detraux, X. Gonze, A. Bongiorno and A. Pasquarello, Phys. Rev. Lett. 89, 117601 (2002).

21 Z.G. Soos and S. Ramasesha, J. Chem. Phys. 90, 1067 (1989).

22 L. Del Freo, A. Painelli and Z.G. Soos, Phys. Rev. Lett. 89, 27402 (2002).

23 Z.G. Soos and D.J. Klein, in Treatise on Solid-State Chemistry, N.B. Hannay, ed. (Plenum, New York, 1976), Vol. III, p. 689 .

24 Z.G. Soos and G.W. Hayden, in Electro-responsive Polymeric Systems T.A. Skotheim, ed. Marcel Dekker, New York, 1988, p.197.

25 Z.G. Soos and S. Ramasesha, in Valence Bond Theory and
Chemical Structure, D.J. Klein and N. Trinajstic, eds. Elsevier, Amsterdam,1989, p. 81; S. Ramasesha and Z.G. Soos, in Theoretical and Computational Chemistry, Vol. 10, D.L. Cooper, ed. Elsevier, Amsterdam, 2002, p. 635.

26 S.P. Karna and A.T. Yates, eds. Nonlinear Optical Materials: Theory and Modeling, ACS Symposium Series 628 (American Chemical Society, Washington, D.C., 1996); G.P. Agrawal, C. Cojan and C. Flytzanis, Phys. Rev. B 17, 776 (1978).

27 F.C. Spano and Z.G. Soos, J. Chem. Phys, 99, 9265 (1993).

28 P.J. Strebel and Z.G. Soos, J. Chem. Phys. 53, 4077 (1970); Z.G. Soos and S. Mazumdar, Phys. Rev. B 18, 1881 (1978).

29 Y. Anusooya-Pati, Z.G. Soos and A. Painelli, Phys. Rev. B 63, 205118 (2001).

30 T. Egami, S. Ishihara and M. Tachiki, Science 261, 1307 (1993); T. Egami and M. Tachiki, Phys. Rev. B 49, 8944 (1994).

31 A. Girlando and A. Painelli, Phys. Rev.B 34, 2131 (1986); A. Painelli and A. Girlando, Phys. Rev.B 37, 5748 (1988).

32 A.J. Heeger, S. Kivelson, J.R. Schrieffer and W.P. Su, Rev. Mod. Phys. 60, 81 (1988).

33 D. Jerome and H.J. Schulz, Adv. Phys. 31, 299 (1982); Low-Dimensional Conductors and Superconductors, NATO-ASI B, 155, D. Jerome, L. Caron eds, Plenum, New York (1987); S. Kagoshima, H. Nagasawa,T. Sambongi, One-dimensional Conductors, Springer-Verlag, Berlin (1988).

34 M.J. Rice and E.J. Mele, Phys. Rev. Lett. 49, 1455 (1982).

35 A. Painelli and A. Girlando, J.Chem.Phys. 87, 1705 (1987).

36 H.M. McConnell, B.M. Hoffmann, R.M. Metzger, Proc.Natl.Acad.Sci. USA 53, 46 (1965); P.L. Nordio, Z.G. Soos, H.M. McConnell, Ann. Rev. Phys. Chem. 17, 237 (1966).

37 M. Masino, A. Girlando and Z.G. Soos, Chem.Phys. Lett. 369, 428 (2003).

38 B. Horovitz and J. Solyom, Phys.Rev.B 35, 7081 (1987).

39 L. Del Freo, F. Terenziani and A. Painelli, J.Chem.Phys. 116,755 (2002).

40 Z.G. Soos, S. Kuwajima and R.H. Harding, J. Chem. Phys. 85,601 (1986);

41 M.J. Rice, Solid State Commun. 31, 93 (1979).

42 A. Girlando, R. Bozio and C. Pecile, Phys. Rev. B, 26, 2306 (1982).

43 A. Painelli and A. Girlando, J. Chem. Phys. 84, 5655 (1986); C. Pecile, A. Painelli and A. Girlando, Mol. Cryst. Liq. Cryst. 171, 69 (1989).

44 R. Bozio and C. Pecile, in Spectroscopy of Advanced Materials, Adv. Spectrosc. Vol. 19, (R.J.H. Clark and R.E. Hester, eds.) Wiley, New York, 1991, p. 1.

45 A. Painelli, L. Del Freo and Z. G. Soos, Synth. Metals, 133-134, 619 (2003).

46 A. Girlando, A. Painelli, S.A.Bewick and Z.G.Soos, Synth. Metals, in press. 